Ann. Sci. forest., 1980, 37 (4), 333-343.

\title{
Die Bedeutung anatomischer Veränderungen \\ bei der Buchenrindennekrose
}

\author{
S. FINK U. H. J. BRAUN \\ Instifut für Forstbotanik und Holzbiologie der Universität \\ Bertoldstrasse 17, 7800 Freiburg i. Br. R. F. A.
}

\section{Zusammenfassung}

Der Befall der Buche durch Cryptococcus fagi führt auf Grund der besonderen Anatomie der Buchenrinde zur Entstehung einer pathologischen Borke mit keilförmig blind an den Sklerose-Baststrahlen endenden Innenperidermen. An diesen Schwachstellen kann die Rinde später aufplatzen und es kommt so zu Nekrosen. Neben dieser rein mechanischen Auswirkung des Lausbesatzes scheinen jedoch noch andere Faktoren für die Krankheitsentstehung eine Rolle zu spielen. Der Vergleich mit dem Befall von Abies balsamea durch Dreyfusia piceae sowie die Ergebnisse älterer Arbeiten über anatomische und chemische Veränderungen im Holz erkrankter Buchen legen den Verdacht einer Injektion von wuchshormon-ähnlichen Substanzen durch die Läuse nahe. Durch Applikation von 0,3-1 p. 100 iger Lösung des Wuchsstoffes 2,4,5-T auf die Rinde älterer Buchen konnten die typischen Symptome der Buchenrindennekrose künstlich induziert werden.

Zur Frage der Schleimflußentstehung wird auf die verschiedenarłigen Ursprungsmöglichkeiten aus dem Holz, dem Phloem und dem geschädigten äußeren Rindengewebe hingewiesen. Verschiedene natürliche und künstliche pathologische Reize können dabei möglicherweise unterschiedliche Arten von «Schleimflu $\beta$ " hervorrufen. Der Untersuchung möglicher aktiver Beteiligung von Mikroorganismen sollte dabei besondere Aufmerksamkeit zugewandt werden.

\section{1. - Anatomische Veränderungen im Rindenbereich}

Die Rinde der Rotbuche (Fagus sylvatica) zeichnet sich dadurch aus, da $\beta$ das Oberflächenperiderm in der Regel während der gesamten Lebensdauer des Baumes als äußerste Schutzschicht erhalten bleibt, d. h. es wird normalerweise keine Borke ausgebildet. Während andere Baumarten mit gleichfalls langlebigem Oberflächenperiderm (z. B. Abies $a l b a$ ) im höheren Alter oder nach äußerlicher Verletzung durchaus eine normale Borke bilden können, ist dies bei der Buche nicht ohne weiteres möglich auf Grund einer einzigartigen anatomischen Besonderheit : dem Besitz von sklerenchymatischen Baststrahlen. Während die Masse der Baststrahlen niedrig, nur wenige Zellschichten breif und parenchymatisch ist, existieren daneben zahlreiche breite, sehr hohe und verholzte Baststrahlen größtenteils primären Ursprungs, die weit in den Rindenbereich hineinragen. Wird nun durch einen äußeren pathologischen Reiz die Bildung eines Innenperiderms als Schułzreaktion erzwungen, so kann dieses nicht durchgehend ausgebildet werden, da im Bereich der Sklerose-Baststrahlen keine Phellogenbildung stattfinden kann. Die Folge ist die Entstehung einer 


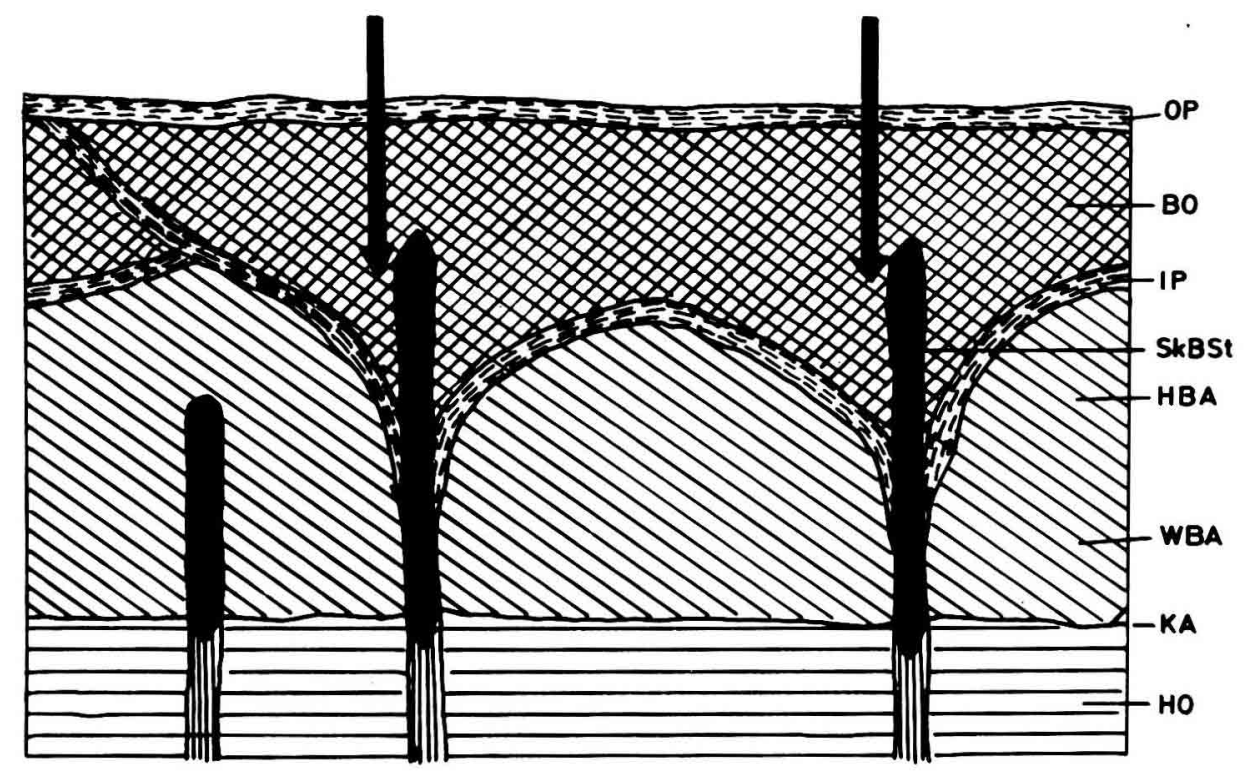

ABB. 1. - Theoretischer Verlauf einer erzwungenen Borkenbildung bei Fagus silvatica L. : Entstehung einer pathologischen Borke (Querschnitts-Schema. OP = Oberflächenperiderm ; BO = Borke ; IP = Innenperiderm SkBST $=$ Sklerose-Baststrahlen ; HBA $=$ Hartbast ; WBA $=$ Weichbast ; KA $=$ Kambium ; $\mathrm{HO}=\mathrm{Holz}$ ). An mit Pfeilen markierten Stellen kann die tote Borke bei zunehmender Spannung infolge des Dickenwachstums leicht aufreißen. (Aus. Braun, 1976).

FIG. 1. - Schéma théorique de formation d'écorce induite chez Fagus silvatica $L$ : Formation d'une écorce pathologique (Schéma de la coupe. OP : périderme externe ; OB : écorce ; IP : périderme interne ; HBA : liber ; WBA : phloème ; KA : cambium ; HO : xylème). Aux endroits indiqués par les flèches l'écorce peut craquer facilement, quand la tension augmente suite à la croissance radicale (d'après Braun, 1976).

pathologischen Borke mit keilförmig blind an den Seiten der Sklerose-Baststrahlen endenden Innenperidermen (Abb. 1). Gerät die Rinde im Verlauf des weiteren Dickenwachstums nun unter tangentiale Zugspannung, so reißt die pathologische Borke an diesen Schwachstellen entlang der Sklerose-Baststrahlen auf und es können so bis zum Kambium reichende Nekrosen entstehen (Braun, 1976, 1977).

Während also z. B. die Weißtanne auf einen äußeren Befall durch parenchymsaugende Rindenläuse (Dreyfusia piceae) mit vollständiger Abriegelung der Befallsstelle mittels eines durchgehenden Innenperiderms reagieren und damit den Schädlingsangriff zum Abklingen bringen kann (Kloft, 1955), so führt eine solche Reaktion der Buche auf Befall durch Cryprococcus fagi nur bei geringflächigem Besatz zum Erfolg ; bei stärkerem Lausbefall wird jedoch auf Grund der oben geschilderten Mechanismen zwangsläufig die Bildung einer pathologischen Borke induziert, die schließlich zu Rindennekrosen führt. Die weitere Ausbreitung der Nekrosen kann dabei durchaus erst zu einem Zeitpunkt augenscheinlich werden, zu dem von dem auslösenden Lausbefall nur noch wenig zu sehen ist. Das Eindringen von Pilzen in die nekrotischen Stellen sowie verstärkte Rindenspannungen in Folge von Witterungsextremen mögen den Krankheitsverlauf dabei beschleunigen.

Die Annahme einer rein mechanischen Wirkung der Einstiche von $C$. fagi im Hinblick auf die Induzierung einer pathologischen Borke mit ihren Folgeerscheinun- 
gen kann das Krankheitsbild der Buchenrindennekrose allein jedoch nicht befriedigend erklären. Vor 2 Jahren mittels feinster Nadelstiche verletzte Buchenrinden zeigen bislang zwar vergleichbare anatomische Veränderungen und Nekrosen wie nach Lausbefall, doch trat kein Schleimfluß auf. Bemerkenswert sind in diesem Zusammenhang auch Beobachtungen an der Rinde von Buchen in den Hochlagen des südlichen Schwarzwaldes, die frei von $C$. fagi sind : Unter dem Einfluß der hohen Feuchtigkeit und des damit verbundenen reichlichen Besatzes der Rinde mit Flechten, Moosen, epiphytischen Pilzen, Bakterien etc. wird eine z. T. recht ausgeprägte Borke ausgebildet, die durchaus ähnliche Struktur wie die pathologische Borke nach Lausbefall aufweist (Abb. 2). Trotz dieser starken Rindenbelastung und des unter den

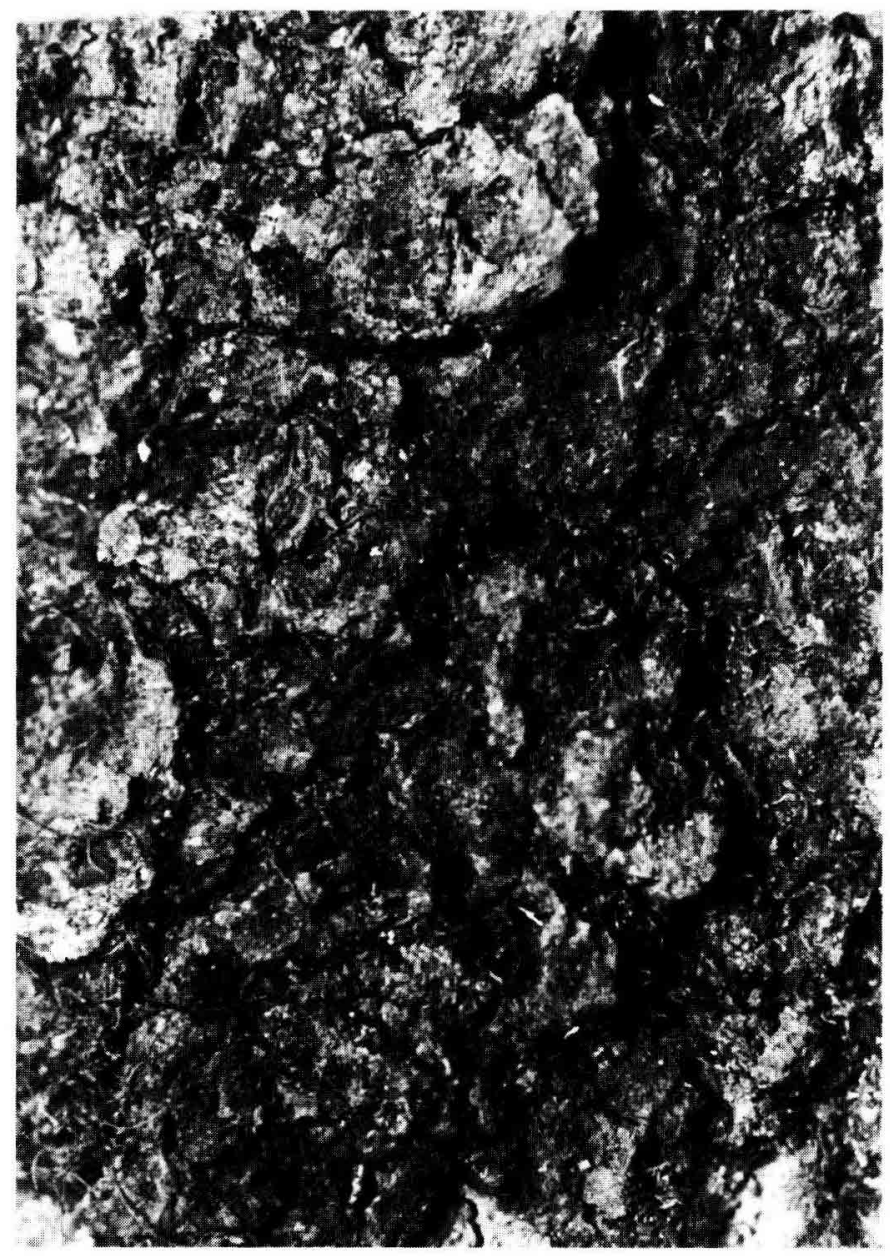

ABB. 2. - Oberfläche einer Buchenrinde aus dem Hochschwarzwald (Schauinsland, ca. $1200 \mathrm{~m}$, ca. $1900 \mathrm{~mm}$ Jahresniederschlag). Nach Entfernung des üppigen Moos- und Flechtenbewuchses zeigt sich eine rauhe, rissige echte Borke.

FIG. 2. - Surface d'une écorce de hêtre d'un peuplement d'altitude en Forêt Noire (Schaninsland, altitude environ $1200 \mathrm{~m}$; précipitations annuelles environ $1900 \mathrm{~mm}$ ). Après enlèvement d'une abondante couche de mousses et de lichens on voit une vraie écorce rugueuse et fissurée. 
feuchten Bedingungen sicher heftigen mikrobiellen Angriffes konnten in diesen Lagen bislang keine Symptome einer Rindennekrose beobachtet werden. Dies mag einmal durch die geringere Wachstumsrate und somit geringeren Zugspannungen in der Rinde erklärbar sein, zum anderen scheint jedoch auch diese Beobachtung darauf hinzudeuten, daß offensichtlich $C$. fagi neben der rein mechanischen Induktion der anatomischen Veränderungen noch durch einen zusätzlichen pathogenen Einfluß die typischen Symplome der Buchenrindennekrose herborruft.

\section{2. - Vergleich mit der Wirkung anderer rindensaugender Stammläuse}

Daß ein Befall durch parenchymsaugende Rindenläuse auch ohne Vorliegen der speziellen anatomischen Reaktion der Buchen rinde ein Absterben von Bäumen hervorrufen kann, davon zeugł z. B. der letale Einfluß von Matsucoccus pini an der Stammrinde von Pinus silvestris (Siewniak, 1970) bzw. von M. feytaudi an P. pinaster (Carle u. Ponty, 1968 ; Carle et al., 1970 ; Carle u. Schvester, 1975). Hier führt tiefe, bis an das Kambium reichende Schutzperidermbildung zu Nekrosen im Siebröhrenbereich («braune Flecken»).

Besonders interessant ist nun ein Vergleich mit den enormen Schäden nach Einschleppung von Dreyfusia piceae nach Nordamerika («balsam wooly aphid»), der gegenüber unsere heimische Abies alba auf Grund der schon oben dargestellten schnell einsetzenden Befallsisolierung durch Bildung eines Innenperiderms zwar resistent ist, auf die die amerikanischen Tannen ( $v$. a. Abies balsamea) jedoch außerordentlich empfindlich reagieren (Carroll u. Bryant, 1960 ; Page, 1975 ; Schooley, 1976). Die langsamere und schwächere Abwehrreaktion ermöglicht den Läusen hier eine längere Einwirkungsdauer (Kloft, 1955). Dadurch gelangen Sekrete bis zum Kambium und es treten unter diesem Einflu $\beta$ anatomische Veränderungen im neugebildeten Holzkörper auf («Rotholzbildung»), die die Wasserleitung behindern und schließlich zum Tod des Baumes führen (Doerksen u. Mitchell, 1965 ; Mitchell, 1967 ; Smith, 1967). Diese Reaktion des Baumes wird möglicherweise durch eine von den Läusen ausgeschiedene Substanz mit wuchshormonähnlichem Charakter ausgelöst (Balch ef al., 1964).

\section{3. - Über den möglichen Einflu $\beta$ von Wuchsstoffen}

Hinsichtlich der Buchenrindennekrose vermutete schon Guillemain (1961 auf Grund seiner Untersuchungen über abweichende Spurenelement-Konzentrationen im Holz erkrankter Buchen eine Injektion von wuchshormon-ähnlichen Speichelbestandteilen durch $C$. fagi. Nach eigenen, noch nicht abgeschlossenen Versuchen konnten die typischen Symptome der Buchenrindennekrose durch Applikation einer 0,3-1 p. 100 igen Lösung von 2, 4, 5-T in Dieselöl auf die Rinde von 50-80-jährigen Buchen künstlich induziert werden. Bei Aufbringung im April und Mai zeigten sich 
nach etwa 2 Monaten die klassischen Krankheitserscheinungen (Nekrosen, Schleimfluß) (Abb. 3). Im Holzkörper bildete sich unter der Einwirkung des Wuchsstoffes eine ähnliche ungeordnet parenchymatische Struktur wie dies Jacquiot (1961) als

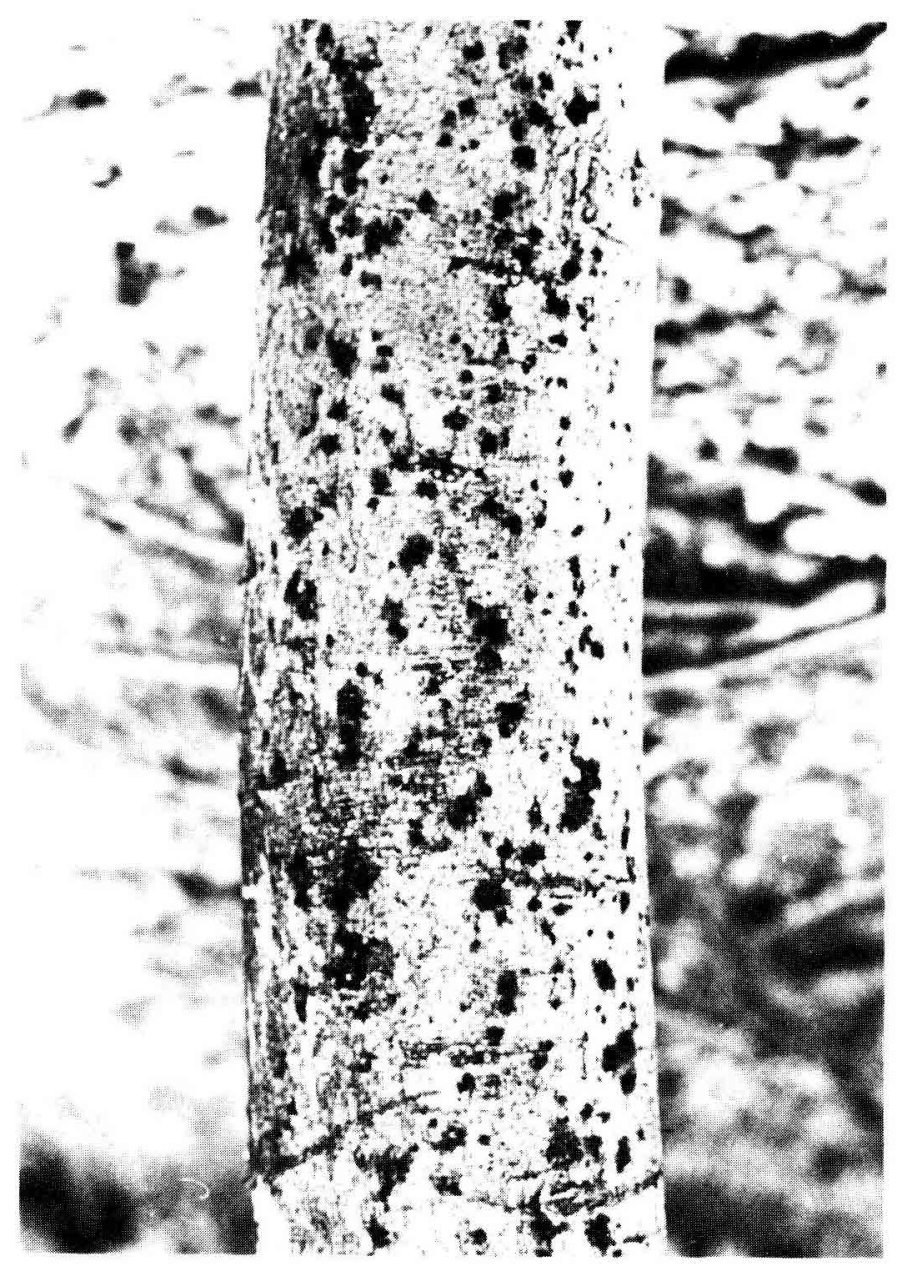

ABB. 3. - Auftreten typischer Schleimflußflecken 2 Monate nach Behandlung der Rinde einer ca. 50-jährigen Buche mit 2,4,5-T im Mai.

FIG. 3. - Apparition de taches suintantes typiques au mois de mai sur un hêtre d'environ 50 ans, 2 mois après traitement au 2-4-5 T.

Symptom der natürlichen Buchenrindennekrose feststellte (Abb. 4). Die Nekrosen reichen z. T. bis zum Kambium, z. T. (besonders bei schwächerer Konzentration des Wuchsstoffes) bleiben sie jedoch auf die äußere Hälfte der Rinde beschränkt, doch konnte auch in diesen Fällen typischer Schleimflu $\beta$ beobachtet werden (Abb. 5). 


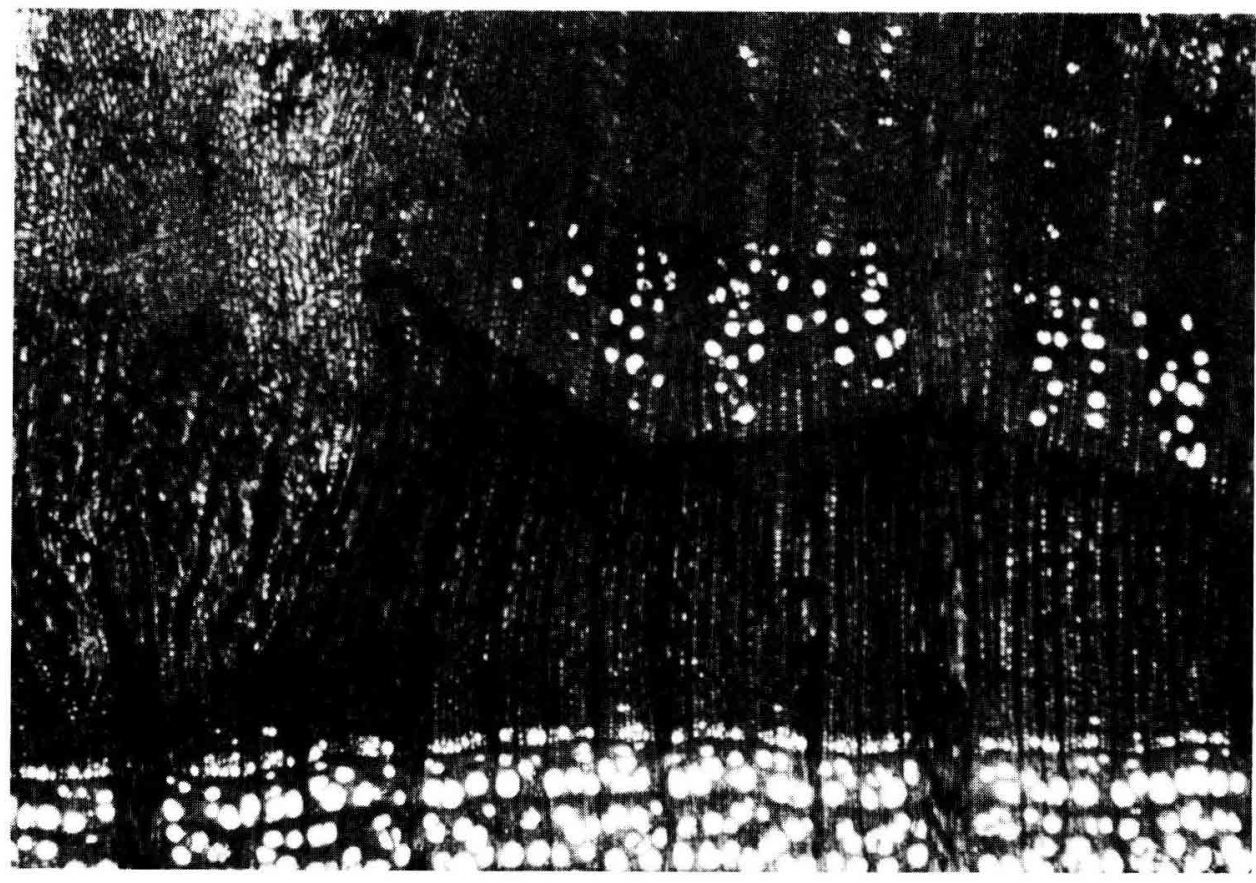

ABB. 4. - Mikroskopischer Querschnitt durch den jünsten Holzteil einer vor 2 Jahren mit 2,4,5-T behandelten Buche. Deutlich erkennbar die plötzliche Umstellung auf Bildung von parenchymatischem Gewebe ohne Gefäße ( $p G)$; erst im zweiten Jahr beginnt sich die Holzbildung mit einigen verstreuten Gefäßen wieder zu normalisieren $(\mathrm{H} 2)$. Auffallend das starke hypertrophe Vermehrungswachstum der Parenchymzellen im Bereich des breiten Holzstrahles (hP). Vgl. dazu Jacquiot (1961).

FIG. 4. - Coupe microscopique de la zone externe du xylème d'un hêtre traité, il y a 2 ans, au 2-4-5 T. On y voit clairement la formation soudaine de tissus parenchymateux sans vaisseaux ( $p G)$; ce n'est que la deuxième année que la formation du xylème commence à se normaliser avec l'apparition de quelques vaisseaux dispersés $(\mathrm{H} 2)$. On observe notamment une forte croissance hypertrophique de cellules de parenchyme près des rayons ligneux larges (hP). (Voir aussi Jacquiot, 1961.)

\section{4. - Zur Schleimfluß-Frage}

Nun handelt es sich bei dem Auftreten von Schleimfluß zweifellos um ein relativ unspezifisches Symptom im Rahmen der Buchenrindennekrose, da ähnliche Erscheinungen auch an anderen Laubhölzern bei verschiedenen Erkrankungen nachgewiesen wurden (Stautz, 1931 ; von Freier, 1960 ; Conrad, 1964 ; Bortitz u. Breuel, 1967 ; Urosevic, 1967 ; Zycha, 1968) ; nach eigenen Beobachtungen konnte sogar an der Weißtanne Schleimfluß nach Verletzungen festgestellt werden. Daß Schleimflu $\beta$ an der Buche experimentell auf verschiedene Weise induziert werden kann, zeigt die Arbeit von Dimitri (1967), der durch Uberhitzung und Unterkühlung der Rinde gleichfalls entsprechende Symptome hervorzurufen vermochte. Auch Befall der Rinde geschwächter Buchen durch Käfer (Agrilus viridis, Taphrorhychus bicolor, Trypodendron domesticum) führf gelegentlich zu Schleimfluß. Jedoch zeigł sich eine solche Reaktion keineswegs als Folge jeder Art von Verletzung oder Rindenabtötung. 


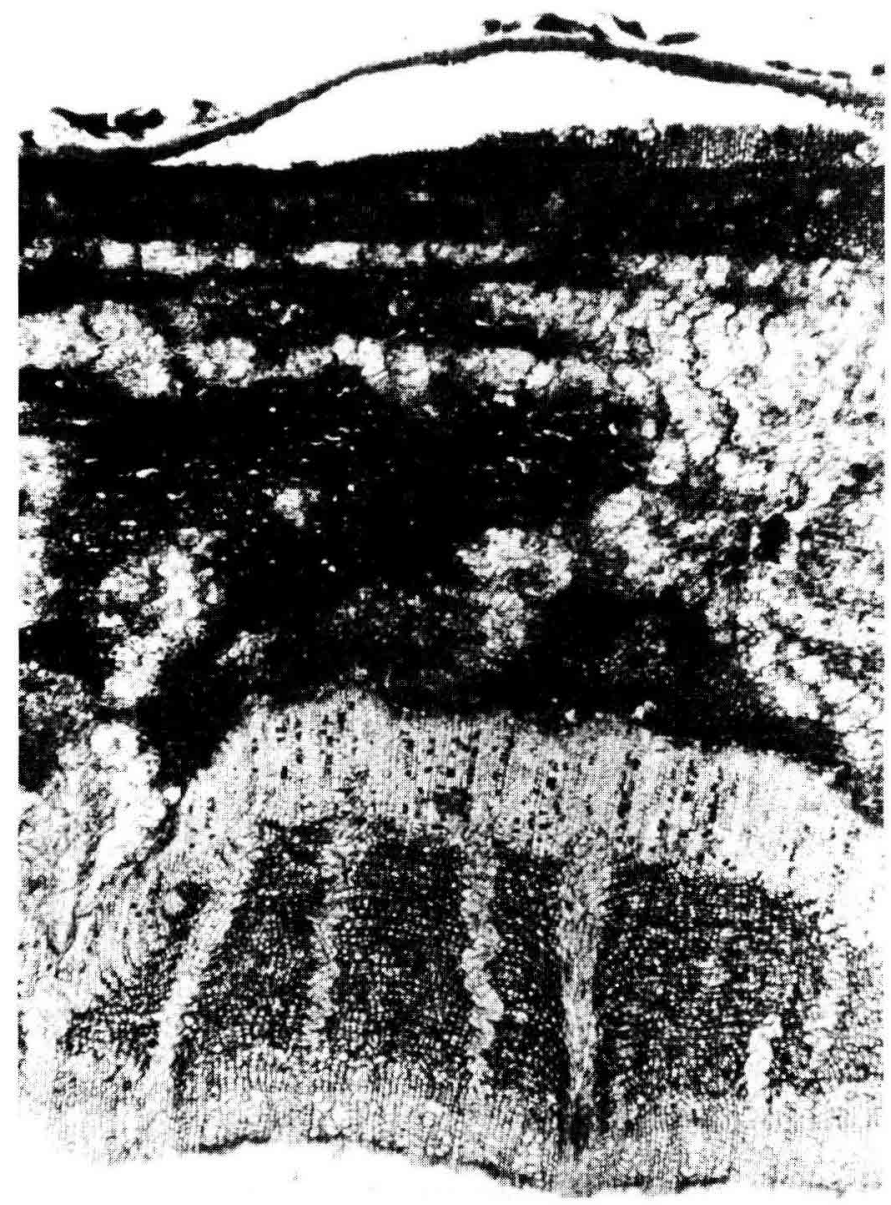

ABB. 5. - Mikroskopischer Querschnitt durch einen Schleimflußflecken aus Abb. 3. Nur der äußere Teil der Rinde ist nekrotische (nR). Gegen das gesunde innere Phloem (gPh) wurde der folte Teil durch ein Innenperiderm (IP) mit mächtif entwickeltem Phelloderm abgeriegelt.

FIG. 5. - Coupe microscopique d'une tache suintante de la figure 3. Seule la partie externe de l'écorce est nécrosée $(\mathrm{nR})$. L1 partie nécrosée est déparée du liber sain $(\mathrm{gPh})$ par un périderme interne (IP) qui comporte un phelloderme fortement développé.

Über den Ursprung des Schleimflusses herrscht noch wie vor noch aine gewisse Unklarheit. Reicht die Nekrose bis zum Kambium, so ist der Ursprung aus dem Xylemwasser des Holzkörpers (besonders während der frühjahreszeitlichen Mobilisationsphase) und dem Phloem (Assimilatstrom) erklärlich (Braun, 1977, Abb. 6), Beschränkł sich das nekrotische Gewebe jedoch auf den äußeren Rindenteil (s. o.), so muß die Entstehung in siłu in dem absterbenden Gewebe angenommen werden (« Reaktionssaft » nach Bortitz u. Brevel, 1967). Inwieveit dabei Mikroorganismen aktiv bei der Schleimflußentstehung beteiligł sind, bedarf noch genauerer Klärung. Eigene Beobachtungen, nach denen die Infektion einer künstlichen Rindenwunde an einer jungen Buche durch einen offenbar holzzersetzenden Basidiomyceten zur Induktion 


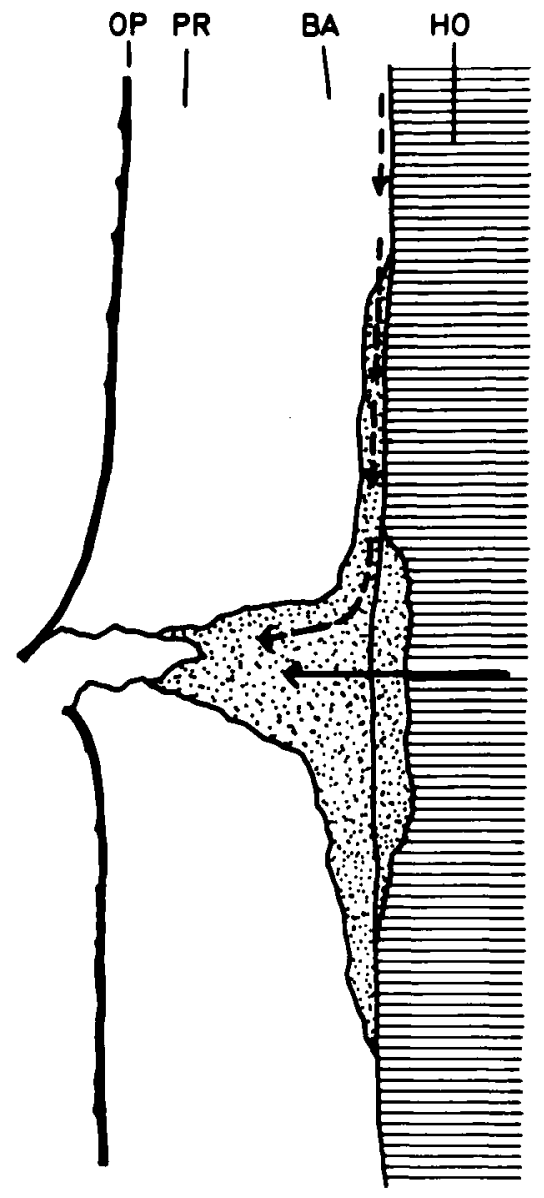

ABB. 6. - Die Möglichkeiten des Saftflusses bei bis zu Kambium reichender Nerkose : 1. aus dem Holz (ausgezogene Pfeile) im zeitigen Frühjahr ; 2. aus dem Leitbast (gestrichelte Pfeile) in der Wachstumszeit oder im Herbst. $(O P=$ Oberflächenperiderm, $P R=$ Pathologische Rinde, BA $=$ Bast, HO $=$ Holz). (Aus Braun 1977).

FIG. 6. - Les possibilités de flux de sève quand les nécroses arrivent jusqu'au cambium : 1. à partir du xylème (flèche à trait plein) ou début du printemps ; 2 à partir du phloème (flèche à firet) pendant la période de croissance ou en automne. ( $O P=$ périderme externe, $P R=-$ écorce nécrosée, $B A=$ phloème et liber, HO $=$ xylème). (D'après Braun, 1977.)

eines sehr dunklen, zähflüssigen Schleimflusses führte, verstärken den Verdachł eines möglichen direkten Einflusses von Mikroorganismen (Abb. 7).

Die oben angeführie neue Methode der künstlichen Erzeugung von Schleimfluß an Buche eröffnet auf Grund der genauen zeitlichen Vorhersagemöglichkeit des Auftretens der Symptome nach der Applikation des Wuchsstoffes die Gelegenheit zur Verfolgung der mikrobiellen Besiedelung des Gewebes schon bevor deutlich sichtbare Reaktionen und damit meisł zugleich auch vielfältige Sekundärbesiedler auftreten. Insbesondere die Rolle von Bakterien in diesem Krankheitskomplex, die 


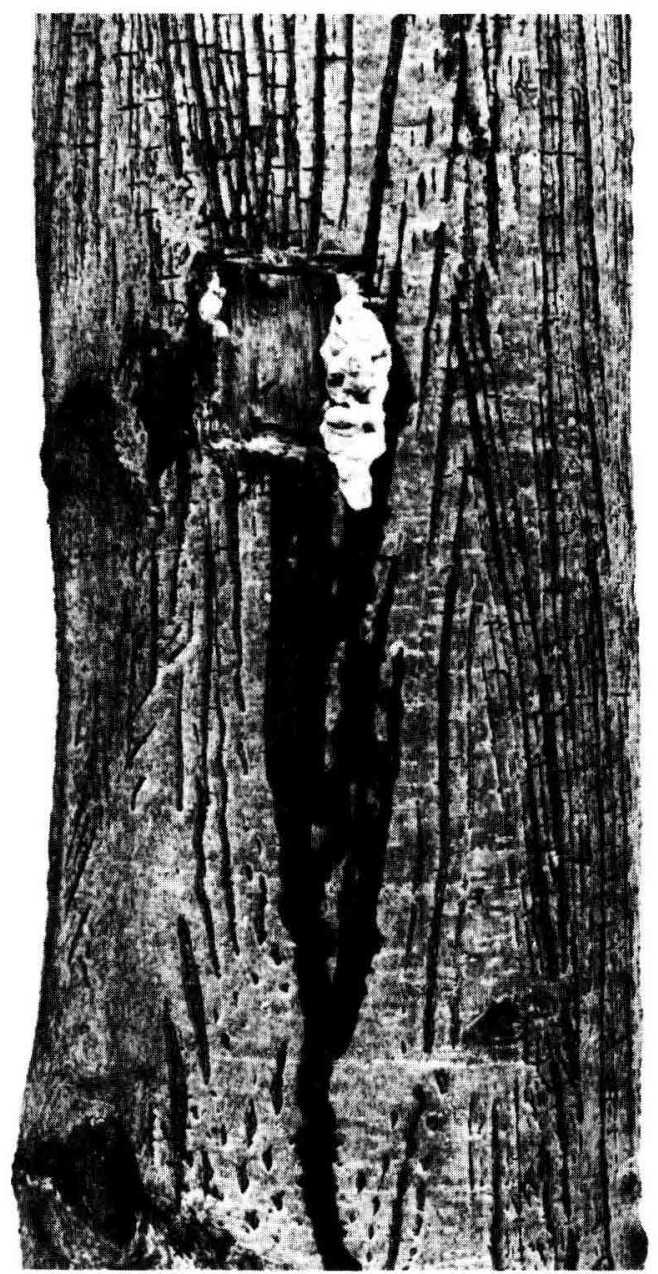

ABB. 7. - Auftreten von dunklem, zähflüssigen Schleimfluß nach Pilzinfektion an einer Entnahmestelle von Rindenproben.

FIG. 7. - Apparition d'un suintement foncé et visqueux après infection fongique à l'endroit de prélèvement d'échantillons de l'écorce.

etwa vor allem von Urosevic (1967) betont wird, könnte dadurch einer Klärung näher gebracht werden.

Abschließend sei jedoch noch darauf hingewiesen, da $\beta$ farbliche und konsistenzmäßige Unterschiede innerhalb natürlich auftretender und künstlich induzierter Schleimflüsse darauf hindeuten, daß unterschiedliche Entstehungsmechanismen durchaus zu verschiedenen Arten von Schleimflu $\beta$ führen können, so da $\beta$ die Notwendigkeit einer genaueren Differenzierung hinsichtlich des umfassenden Phänomens «Schleimflu $\beta$ » bestehen wird. 


\title{
Résumé
}

\section{L'importance des changements anatomiques pour l'étiologie et pour la symptomatologie} du dépérissement du hêtre ainsi que quelques remarques sur le problème

\author{
du suintement
}

\begin{abstract}
Une écorce pathologique se différencie à la suite des attaques de Cryplococcus fagi, présentant des péridermes intérieurs jusqu'aux rayons libériens sclérosés en forme de coin. Si l'écorce éclate à proximité des points d'attaques, il se forme une nécrose. En plus des effets strictements mécaniques, d'autres facteurs jouent un rôle important dans l'origine de la maladie. La comparaison avec les attaques de Dreyfusia picea sur Abies balsamea et les précédentes recherches sur les changements anatomiques ef chimiques du bois des hêtres atteints suggèrent que la cochenille injecte dans le bois des substances comparables aux substances de croissance. L'application d'une substance de croissance 2,4,5-T (0,31 p. 100) sur les écorces de vieux hêtres induit artificiellement les symptômes typiques du dépérissement du hêtre. Nous voulons attirer l'attention sur les multiples origines des suintements : xylème, phloème et écorce externe. Il est possible que les divers événements pathologiques naturels ou artificiels provoquent différentes sortes de suintement. Dans ces recherches, il fout porter une attention toute particulière à la participation active des micro-organismes.
\end{abstract}

\section{Summary}

\section{The significance of anatomical alterations for the etioloqy and symptomatology of Beech bark disease and some remarks on the problem of slime-flux}

Due to the special anatomy of beech bark, the infestation of the beech by Cryptococcus fogi leads to the formation of a pathological rhytidome with wedge-shaped, interior peridems ending abruptly at the sclerotic phloem rays. The bark may crack at these weak points and necroses may arise. Nevertheless it seems that as well as this mechanical effect of the coccid attack, there are some other factors involved in the development of the disease. The comparison with the infestation of Abies balsamea by Dreyfusia piceae, as well as the results of previous investigations on anatomical and chemical changes in the wood of diseased beech trees, lead to the suspicion that the coccids may inject growth-hormone-like substances. By application of 0.3-1 p. 100 solution of the growth-regulator 2,4,5-T on to the bark of older beeches the typical symptoms of beech bark disease could be induced artificially. With regard to the problem of slime-flux, the different possibilities of origin from the $x y l e m$, the phloem and the diseased outer bark tissues are mentioned. Various naturally occuring and artificially induced pathological factors may lead to different kinds of "slime-flux ". In this connection special attention should be drawn to the possible active contribution of microorganisms.

\section{Références bibliographiques}

BALCH R. E., CLARK J. u BONGA J. M., 1964. Hormonal action in production of tumours and compression wood by an aphid. Nafure, 202, 721-722.

BÖRTITZ S.u. BREUEL K., 1967. Beiträge zur Ätiologie des Braunfleckengrindes, einer Rindennekrose der Gattung Populus. 3. Mitt. : Untersuchungen über die Flüssigkeit im Schadbereich (« Reaktionssaft »). Phyfopath. Z., 58, 281-290.

BRAUN H. J., 1976. Das Rindensterben der Buche, Fagus sylvatica L., verursacht durch die Buchenwollschildlaus Cryptococcus fogi Bär. I. Die Anatomie der Buchenrinde als Basis-Ursache. Eur. J. For. Path., 6, 136-146.

BRAUN H. J., 1977. Das Rindensterben der Buche, Fagus sylvafica L., verursacht durch die Buchenwollschildlaus Cryptococcus fagi Bär. Il. Ablauf der Krankheit. Eur. J. For. Path., 7, 76-93.

CARLE P. u. de PONTY G. R., 1968. Le dépérissement du Pin maritime dans le var - épidemiologie - symptomatologie - cause primaire. Rev. for. frone., 20, 185-203.

CARLE P. u., SCHVESTER P., 1975. Perspectives d'avenir du pin marifime en provence (Pinus pinaster Ait. var. mesogeensis Fieschi et Gaussen). Rev. for. franc., 27, 339-349.

CARLE P., CHARDE J.-P. u. BOULAY C., 1970. Comportement de piqûre de Matsucoccus feytaudi Duc. (Coc., Margarodidae) ; caractérisation histologique et histochimique des désorganisations engendrées dans le végétal (Pinus pinoster Ait. var. mesogeensis). Ann. sci. forest., 27, 89-104. 
CARROLL W. J. U. BRYANT D. G. 1960. A review of the balsam wooly aphid in Newfoundland. For. Chron., 36, 278-290.

CONRAD J. 1964. Schleimflußkrankheit auch beim Spitzahorn. Allg. Forstzeitschr., 19, 730.

DIMITRI L., 1967. Untersuchungen über die Ätiologie des « Rindensterbens » der Buche. Forsfwiss. Cbl., 83, 257-276.

DOERKSEN A. H. U., MITCHELL R. G., 1965. Effects of the balsam wooly aphid upon wood anatomy of some western true firs. For. Sci., 11, 181-188.

FREIER R. von, 1960. Schleimflußkrankheit an Birke und anderen Holzarten. Forst- u. Holzwirt, 16, 55-56.

GUILLEMAIN J., 1961. Etude d'une maladie du hêtre ou de fault du « T ». Ann. Ec. Eaux For. Nancy, 18, 647-659.

JACQUIOT C., 1961. Note préliminaire sur une maladie du bois de hêtre dans l'est de la France. Rev. for. franc., 13, 167-170.

KLOFT W., 1955. Untersuchungen an der Rinde von Weibtannen (Abies pectinata) bei Befall durch Dreyfusia (Adelges) piceae Ratz. Z. angew. Entomol., 37, 340-348.

MITCHELL R. G., 1967. Abnormal ray tissue in three true firs infested by the balsam wooly aphid. For. Sci., 13, 327-332.

PAGE G., 1975. The impact of balsam wooly aphid damage on balsam fir stands in Newfoundland. Can. J. For. Res., 5, 195-209.

SCHOOLEY H. O., 1976. Recovery of young balsam fir trees damaged by balsam wooly aphid. For. Chron., 62, 143-144.

SIEWNIAK M., 1970. Matsucoccus pini (Margarodidae, Coccoidea) als neuer weitverbreiteter SchädIing von Pinus silvestris. Morphologie und Bionomie des Schädlings, Art und Umfang der Schädigung. Dissertation Dresden, $129 \mathrm{~S}$. +55 Abb. - 26 Tab.

SMITH F. H., 1967. Effects of valsam wooly aphid (Adegfes piceae) infestation on cambial activity in Abies grandis. Amer. J. Bot., 54, 1215-1223.

STAUTZ W., 1931. Beiträge zur Schleimflußfrage. Phytopath. Z., 3, 163-229.

UROSEVIC B., 1967. Weeping necroses of forest trees found on the territory of Czechoslovakia. XIV. IUFRO-KONGRESS München 1967, V, Sect. 24, S. 485-493.

ZYCHA H., 1968. Eine Schleimflußerkrenkung der Roteiche. Allg. Forst- u. Jagizig., 139, 195-197. 